\title{
THYROID
}

\section{Clinical significance of neutrophil-to-lymphocyte ratio, lymphocyte-to-monocyte ratio, platelet-to-lymphocyte ratio and prognostic nutritional index in low-risk differentiated thyroid carcinoma}

\author{
Significato clinico del rapporto neutrofili/linfociti, rapporto linfociti/monociti, rapporto \\ piastrine/linfociti e indice prognostico nutrizionale nei carcinomi differenziati \\ della tiroide a basso rischio
}

\author{
Chiara Offi, Roberto Maria Romano, Angelo Cangiano, Giancarlo Candela, Giovanni Docimo \\ Division of Thyroid Surgery, Department of Medical and Advanced Surgical Sciences, University of Campania "Luigi Vanvitelli", \\ School of Medicine, Naples, Italy
}

\begin{abstract}
SUMMARY
Objective. Inflammation and nutritional status play an important role in the prognosis of cancer. Lymphocyte-to monocyte ratio (LMR), neutrophil-to-lymphocyte ratio (NLR), platelet-to-lymphocyte ratio (PLR) and prognostic nutritional index (PNI) are independent prognostic scores in numerous cancers. However, any study showed their prognostic role in low-risk differentiated thyroid carcinoma (DTC). We aimed to clarify and identify the prognostic value of inflammation indices in low-risk DTC patients.

Methods. We analysed data from 116 patients, dividing the population into two groups, according to AJCC staging system ( $8^{\text {th }}$ edition). The LMR, NLR, PLR and PNI cut-off value were determined using receiver operating characteristic (ROC) curve. Disease-free survival (DFS) was calculated with Kaplan-Meyer and Log-Rank tests and the risk of recurrence was calculated with univariate and multivariate Cox regression. Statistical significance was $\mathrm{p}<0.05$.

Results. We found a baseline NLR value $\geq 1.750$ (75\% sensitivity, $40.2 \%$ specificity) and a baseline LMR value of 3.83 (66.7\% sensitivity, $48.9 \%$ specificity). Overall DFS was $74.995 \pm 3.236$ with a $\mathrm{p}$ value of 0.678 . NLR showed a hazard ratio for recurrence with almost twice the risk of recurrence (Adjusted Hazard Ratio /HR ${ }^{\mathrm{A}}$ ): 1.828 , p-value $=0.019$ ).

Conclusions. NLR can be considered a prognostic score with twice the risk of recurrence in low-risk DTC patients with NLR $<1.750$.
\end{abstract}

KEY WORDS: thyroid carcinoma, neutrophil-to-lymphocyte ratio, lymphocyte-tomonocyte ratio, platelet-to-lymphoctye ratio, prognostic nutritional index

\section{RIASSUNTO}

Oggetto. L'infiammazione e lo stato nutrizionale giocano un ruolo fondamentale nella prognosi del cancro. LMR, NLR, PLR, e PNI sono considerati fattori prognostici indipendenti in numerosi carcinomi. Tuttavia, nessuno studio ha dimostrato il loro ruolo nella prognosi dei DTC-basso rischio. L'obiettivo del nostro studio è chiarire e indentificare il valore prognostico di tali valori nei pazienti con DTC-basso rischio.

Metodi. Abbiamo analizzato 116 pazienti, dividendo la popolazione in due gruppi secondo la stadiazione AJCC. Il valore di LMR, NLR, PLR e di PNI è stato determinato con la curva ROC. La DFS è stata calcolata con il test di Kaplan-Meyer e con il test Log-Rank, mentre il rischio di recidiva è stato calcolato con la regressione univariata e multivariata di Cox. La significatività statistica è stata considerata con valori di p-value $<0,05$.

Risultati. Abbiamo trovato un valore di NLR $\geq 1,750$ (75\% sensibilità, 40,2\% specificità)
Received: August 29, 2020

Accepted: October 26, 2020

\section{Correspondence}

Chiara Offi

Department of Medical and Advanced Surgical Sciences, University of Campania "Luigi Vanvitelli", School of Medicine, piazza Miraglia 2, 80138 Naples, Italy

Tel. +390815665275. Fax +390815665257

E-mail: chiara.o@live.com

Funding

None.

Conflict of interest

The Authors declare no conflict of interest.

\begin{abstract}
How to cite this article: Offi C, Romano RM, Cangiano A, et al. Clinical significance of neutrophil-to-lymphocyte ratio, lymphocyteto-monocyte ratio, platelet-to-lymphocyte ratio and prognostic nutritional index in low-risk differentiated thyroid carcinoma. Acta Otorhinolaryngol Ital 2021;41:31-38. https://doi. org/10.14639/0392-100X-N1089
\end{abstract}

() Società Italiana di Otorinolaringoiatria e Chirurgia Cervico-Facciale

\section{c) (1) $(9)$}

This is an open access article distributed in accordance with the CC-BY-NC-ND (Creative Commons Attribution-NonCommercial-NoDerivatives 4.0 International) license. The article can be used by giving appropriate credit and mentioning the license, but only for non-commercial purposes and only in the original version. For further information: https:// creativecommons.org/licenses/by-nc-nd/4.0/deed.en 
e di LMR di 3,83 (66,7\% sensibilità, 48,9\% specificità). La DFS è di 74,995 \pm 3,236 con p-value di 0,678. NLR ha mostrato un Hazard Ratio con un rischio di recidiva doppio $\left(H R^{A} 1,828\right.$, p-value $\left.=0,019\right)$.

Conclusioni. NLR può essere considerato un fattore prognostico con un rischio di recidiva duplicato nei pazienti con DTC-basso rischio con valori di $N L R<1,750$.

PAROLE CHIAVE: carcinoma tiroideo, rapporto neutrofili/linfociti, rapporto linfociti/monociti, rapporto piastrine/linfociti, indice prognostico nutrizionale

\section{Introduction}

Papillary thyroid cancer (PTC) and follicular thyroid cancer (FTC) account for $85 \%$ and $12 \%$, respectively, of all thyroid cancers and are defined as differentiated thyroid carcinomas (DTC) ${ }^{1}$. The incidence of DTC has doubled $(28.2 / 100,000$ new cases per year in women and $10.1 / 100,000$ new cases in men) in the past decade and the increase is more marked than any other cancer ${ }^{2}$. DTC is more frequent in women, but the mortality is similar between genders ( 1 death per year per 100,000 people) ${ }^{3}$. The clinical presentation of DTC varies from an indolent tumour with very low mortality to a very aggressive malignancy ${ }^{3}$. PTC has a predominantly lymphatic spread, while FTC shows haematic spreading ${ }^{2}$. Diagnostic suspicion is confirmed with ultrasonography imaging and echo-guided fine needle cytology (FNC) ${ }^{3}$. According to the American Thyroid Association (ATA), the recurrence rate in low-risk DTC is less $1-2 \%{ }^{3}$. The treatment of choice in DTCs is surgery followed or not by radioactive iodine (RAI) therapy ${ }^{3}$. Pathological tumour-node-metastases (TNM) stage is the major prognostic factor in DTC ${ }^{3}$. The lymph node recurrence rate is variable in low-risk DTC changes according to histotype of the neoplasm and response to surgical treatment and RAI therapy ${ }^{1}$. Therefore, finding indices that can predict the presence of lymph node metastases at diagnosis or the rate of lymph node recurrence in low-risk DTCs can be a valid help in planning tailored treatment (thyroidectomy versus hemithyroidectomy and postoperative RAI therapy).

The immune system is potentially able to eradicate tumours; the inflammatory response is associated with initiation, progression, metastasis and prognosis ${ }^{4}$. Neutrophils, lymphocytes, monocytes and platelets are inflammatory mediators. Neutrophils and platelets product pro-inflammatory cytokines such as endothelial growth factor (VEGF), tumour necrosis factor- $\alpha$ (TNF- $\alpha$ ), interleukin-2 (IL-2), interleukin-6 (IL-6) and interleukin-10 (IL-10), which contribute to tumourigenesis. Monocytes and lymphocytes, on the other hand, have anti-tumoural effects ${ }^{4}$. The literature confirms that the neutrophil-to-lymphocyte ratio (NLR), platelet-to-lymphocyte ratio (PLR) and lymphocyte-tomonocyte ratio (LMR) are independent prognostic factors in many solid cancers, including colorectal cancer, lung cancer, oesophagus cancer and laryngeal squamous cell carcinoma ${ }^{5-7}$. Prognostic nutritional index (PNI) is a new prognostic score that reflects inflammatory and nutritional status of patient ${ }^{8}$. The recent literature shows that PNI is a predictive and prognostic index in many solid tumours, such as colorectal cancer, gastric cancer, malignant pleural mesothelioma, hepatocellular carcinoma, pancreatic cancer and breast cancer ${ }^{9-11}$. All these indices can be calculated on routine preoperative blood samples and biochemical tests, with no additional costs.

NLR, LMR, PLR and PNI, to our knowledge, have never been investigated in patients with low-risk DTC. Therefore, the routine use of these inflammatory prognostic indices might add prognostic stratification in low-risk DTC patients.

According to the 2015 ATA guidelines, low-risk DTC is defined as: PTC without local or distant metastases; PTC with R0 after surgery; PTC without invasion of locoregional tissues; PTC without an aggressive histology; PTC without metastatic foci outside thyroid bed after RAI therapy; PTC without vascular invasion; PTC with clinical NO or with $\leq 5$ pathological micrometastases (N1c); intrathyroidal follicular variant of PTC; intrathyroidal FTC with capsular invasion and no or $<4$ foci of vascular invasion and intrathyroidal papillary unifocal or multifocal microcarcinoma with or without $\mathrm{BRAF}^{\mathrm{V} 600 \mathrm{E}}$ mutations ${ }^{1}$. We have investigated the association between preoperative NLR, PLR, LMR, PNI and the presence of lymph node metastasis and overall survival (OS) and disease-free survival (DFS) in patients with low-risk DTC undergoing or not RAI therapy. Therefore, the aim of this study was to understand the prognostic value of inflammation indices in low-risk DTC patients.

\section{Materials and methods}

We have retrospectively collected data on 208 patients who underwent thyroidectomy or hemithyroidectomy at the Department of Thyroid Surgery at the University of Campania "Luigi Vanvitelli". All patients had an anatomopathological diagnosis of DTC and underwent surgery between March 2012 and December 2019. Clinical and pathological TNM staging was performed according to the American Joint Committee on Cancer (AJCC) staging system $\left(8^{\text {th }}\right.$ edition) ${ }^{12}$. We enrolled 116 patients. 
We included patients aged over 18 years and not pregnant with: single or multiple thyroid nodule with cytological diagnosis of TIR1c, TIR3a, TIR3b, TIR4 and TIR5 to FNC; characteristic of low-risk DTC, according to 2015 ATA guidelines; complete medical history and complete preoperative blood tests. We excluded patients with: presence of single or multiple thyroid nodule with cytological diagnosis of TIR1 or TIR2; other histopathological thyroid cancer as medullary thyroid cancer (MTC) or anaplastic thyroid cancer (ATC); autoimmune thyroid disease (Hashimoto's thyroiditis or Basedow's disease); neck surgery within 5 years; neck radiation therapy; previous thyroid tumour; previous tumour of the head-neck district; chronic inflammation due to HBV, HCV, chronic gastritis, nephritis or gout; immune or haematological disease; history of chronic anticoagulant drugs use and proliferative haemopoietic disorders. The flow chart (Fig. 1) shows the patients enrolled according to the inclusion and exclusion criteria.

We divided the population into two groups based on age: $\leq 55$ years and $>55$ years, according to the AJCC thyroid staging system ( $8^{\text {th }}$ edition). All patients underwent blood and biochemical analyses, FNC, thyroid and laterocervical lymph node ultrasound and fibreoptic-laryngoscopy in the 30 days prior to surgery. Clinical and demographic data as age and sex were obtained from the archive files. Anatomopathological findings suchas histopathological type,

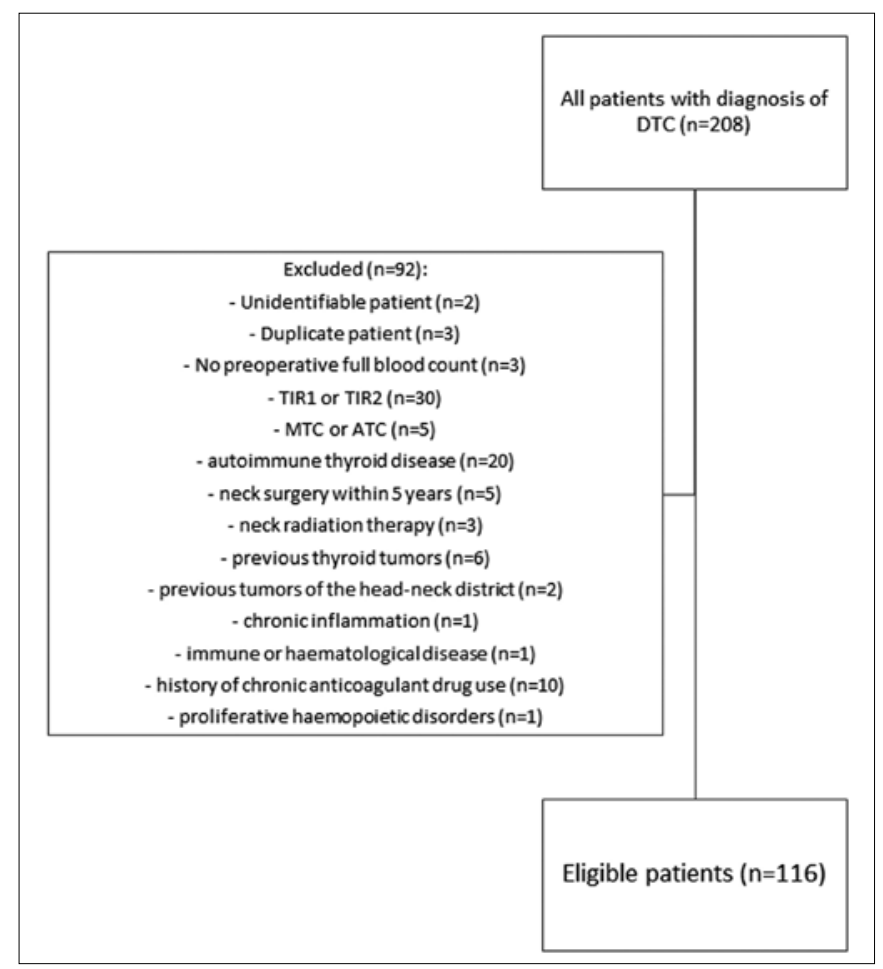

Figure 1. Flow chart of eligible patients. dimension, capsular invasion, vascular invasion, laterality, unifocality, multifocality and stage were obtained from archive files. Laboratory data on white blood cells, neutrophils, lymphocytes, monocytes, eosinophils, basophils and platelets were obtained from archive data. NLR, LMR and PLR were obtained from the simple relationship between the two values; PNI was obtained using the following formula: $[10 \mathrm{x}$ albumin $(\mathrm{g} / \mathrm{dL})+(0.005 \mathrm{x}$ total lymphocytes count $\left.\left(10^{3} / \mu \mathrm{L}\right)\right]$. All surgical procedures were performed by two surgeons. The surgical treatments performed were total thyroidectomy (TT), near total thyroidectomy (NTT) or hemithyroidectomy (HT). TT is the surgical treatment of choice in the treatment of thyroid diseases that requires extracapsular removal of the gland in its entirety. NTT involves the extracapsular removal of the gland leaving a small thyroid residue at the level of the recurrent laryngeal nerve or at the level of the nerve entrance at the laryngeal level where total removal is associated with a sacrifice of the nerve itself. HT involves the removal of the thyroid lobe associated with the removal of the isthmus. These surgical treatments are foreseen in the treatment of DTC by the ATA guidelines ${ }^{1}$.

During follow-up we assessed postoperative RAI therapy $\mathrm{I}^{131}$, the rate of lymph node recurrence and DFS. After primary surgery, a follow-up programme was carried out for all patients. During the first years, physical examination, serum thyroglobulin $(\mathrm{Tg})$ and neck ultrasound were performed every 3 months and every 6 months from the second year. In our population we had a follow-up ranging from 3 to 93 months. Recurrence was defined by increased serum $\operatorname{Tg}(\geq 20 \mu \mathrm{g} / \mathrm{L})$ and the appearance of suspicious lymph node metastases on neck ultrasound, and then confirmed with FNC and Tg measurement in FNC eluates. DFS was defined as the interval between primary surgery and lymph node recurrence.

This study was approved by the Ethics Committee of the University of Campania "Luigi Vanvitelli" (AOU4588/2020). Written informed consent was obtained from all participants. All procedures performed were in according to Helsinki Declaration.

\section{Statistical analysis}

Continuous variables were described as mean and standard deviation (SD), while categorical variables were described as number of cases and percentage. Independent t-tests were performed to compare continuous variables (month of recurrences, maximum diameter of neoplasm and all haematological values with their relatives score), and a test of proportions was applied to categorical variables (number of patients, FNC, RAI, lymph node recurrences and microcarcinoma). The LMR, NLR, PLR and PNI cut-off values 
were determined using a receiver operating characteristic (ROC) curve. Moreover, Fisher's exact test was used to analyse gender, surgical treatment, laterality of neoplasm and histopathological reports. DFS was calculated with the Kaplan-Meyer method and Log-Rank test was used to compare recurrences. The risk of recurrence (Hazard Ratio, HR) was calculated using univariate and multivariate Cox's regression. Statistical significance was considered in case of $p$ value $<0.05$. Statistical analysis was performed with SPSS version 23 (SPSS $^{\odot}$, Chicago, IL, USA).

\section{Results}

We retrospectively enrolled 116 consecutive patients who underwent surgery for a low-risk DTC in our department from March 2012 and December 2019. According to AJCC stage, patients were divided in two groups: low-risk DTC $\leq 55$ years and low-risk DTC $\leq 55$ years. Baseline demographic characteristics, cytology details, surgical treatment and anatomopathological report are shown in Table I. The study cohort included $80(68.9 \%)$ patients $\leq 55$ years and $36(31.1 \%)$ patients $>55$ years; the mean age was 43.1 years in the group $\leq 55$ years and 63.8 years in the group $>55$ years. Female patients were $85.3 \%$ without no significant difference (p-value 0.26 ) between groups. There was no TIR1c in our population. TIR3a, TIR3b, TIR4 and TIR5 in the $\leq 55$ year group were $3.75 \%, 40 \%, 18.75 \%$ and $37.5 \%$, respectively, and $8.34 \%, 25 \%, 30.55 \%$ and $36.11 \%$, respectively, in the $>55$ year group with no significant difference. The principal surgical procedures applied were TT/NTT, and HT was performed in only $5.2 \%$ of the cohort ( $\mathrm{p}=0.08)$. Tumours were present in $41.4 \%$ in the right lobe and in $58.6 \%$ in the left lobe $(\mathrm{p}=0.06)$. We analysed only low-risk DTC and recorded 106 cases $(91.4 \%)$ of PTC and 10 cases $(8.6 \%)$ of FTC $(\mathrm{p}=0.49)$. We found a microcarcinoma in $50 \%$ of the cohort $(\mathrm{p}=0.99)$. In the entire population, we recorded a maximum diameter of neoplasia of $1.37 \mathrm{~cm}(\mathrm{p}=0.403)$. In the $\leq 55$ year group, $100 \%$ of

Table I. Baseline characteristics of patients.

\begin{tabular}{|c|c|c|c|c|}
\hline Characteristic & Population & $\begin{array}{c}\leq 55 \text { years } \\
\text { (according to AJCC stage) }\end{array}$ & $\begin{array}{l}\quad>55 \text { years } \\
\text { (according to AJCC stage) }\end{array}$ & $\mathrm{p}$-value \\
\hline Patients (\%) & 116 & $80(68.9)$ & $36(31.1)$ & $<0.001$ \\
\hline Mean age in years (SD) & $49.2( \pm 12.45)$ & $43.1( \pm 8.45)$ & $63.8( \pm 8.58)$ & $<0.001$ \\
\hline \multicolumn{5}{|l|}{$\operatorname{Sex}(\%)$} \\
\hline Male & $17(14.7)$ & $14(17.5)$ & $3(8.4)$ & \multirow[t]{2}{*}{0.26} \\
\hline Female & 99 (85.3) & $66(82.5)$ & 33 (91.6) & \\
\hline \multicolumn{5}{|l|}{ FNC (\%) } \\
\hline TIR1C & $0(0)$ & $0(0)$ & $0(0)$ & 0.99 \\
\hline TIR3a & $6(5.2)$ & $3(3.75)$ & $3(8.34)$ & 0.56 \\
\hline TIR3b & $41(35.3)$ & $32(40)$ & $9(25)$ & 0.17 \\
\hline TIR4 & $26(22.4)$ & $15(18.75)$ & $11(30.55)$ & 0.24 \\
\hline TIR5 & $43(37.1)$ & $30(37.5)$ & $13(36.11)$ & 0.72 \\
\hline \multicolumn{5}{|l|}{ Surgery (\%) } \\
\hline TT/NTT & $110(94.8)$ & 78 (97.5) & $32(88.9)$ & \multirow[t]{2}{*}{0.08} \\
\hline HT & $6(5.2)$ & $2(2.5)$ & $4(11.1)$ & \\
\hline \multicolumn{5}{|l|}{ Laterality of tumour (\%) } \\
\hline Right & $48(41.4)$ & $29(35)$ & 19 (52.8) & \multirow[t]{2}{*}{0.06} \\
\hline Left & $68(58.6)$ & $53(65)$ & $15(47.2)$ & \\
\hline \multicolumn{5}{|l|}{ Anatomopathological reports (\%) } \\
\hline PTC & $106(91.4)$ & $74(92.5)$ & $32(88.9)$ & \multirow[t]{2}{*}{0.49} \\
\hline FTC & $10(8.6)$ & $6(7.5)$ & $4(11.1)$ & \\
\hline Mean maximum diameter in $\mathrm{cm}(\mathrm{SD})$ & $1.37( \pm 1.89)$ & $1.47( \pm 2.2)$ & $1.15( \pm 0.8)$ & 0.403 \\
\hline \multicolumn{5}{|l|}{ Stage (\%) } \\
\hline 1 & $111(95.7)$ & $80(100)$ & $31(86.1)$ & \multirow[t]{2}{*}{0.002} \\
\hline$\|$ & $5(4.3)$ & $0(0)$ & $5(13.9)$ & \\
\hline Microcarcinoma (\%) & $58(50)$ & $40(50)$ & $18(50)$ & 0.99 \\
\hline
\end{tabular}

SD: standard deviation; PTC: papillary thyroid carcinoma; FTC: follicular thyroid carcinoma; TT: total thyroidectomy; NTT: near total thyroidectomy; HT: hemithyroidectomy. 
Table II. Baseline haematological values.

\begin{tabular}{lcccc} 
Blood values & Population & $\begin{array}{c}\leq 55 \text { years } \\
\text { (according to AJCC stage) }\end{array}$ & $\begin{array}{c}>55 \text { years } \\
\text { (according to AJCC stage) }\end{array}$ & p-value \\
Mean albumin (SD) & $4.49( \pm 0.31)$ & $4.51 \pm 0.26)$ & $4.45( \pm 0.40)$ & 0.356 \\
Mean white blood cells (SD) & $6.82( \pm 2.17)$ & $7.03 \pm 2.18)$ & $6.53( \pm 2.09)$ & 0.116 \\
Mean neutrophils (SD) & $4.37( \pm 1.67)$ & $4.61( \pm 1.74)$ & $3.84( \pm 1.38)$ & 0.21 \\
Mean lymphocytes (SD) & $2.45( \pm 4.29)$ & $2.65 \pm 5.15)$ & $2.02( \pm 0.73)$ & 0.467 \\
Mean monocytes (SD) & $0.42( \pm 0.14)$ & $0.43 \pm 0.14)$ & $0.39( \pm 0.13)$ & 0.157 \\
Mean eosinophils (SD) & $0.16( \pm 0.10)$ & $0.16( \pm 0.10)$ & $0.17( \pm 0.09)$ & 0.549 \\
Mean basophils (SD) & $0.04( \pm 0.03)$ & $0.04 \pm 0.03)$ & $0.03( \pm 0.01)$ & 0.06 \\
Mean platelets (SD) & $259.8( \pm 52.05)$ & $266.08( \pm 51.10)$ & $245.69( \pm 52.09)$ & 0.051 \\
Mean LMR (SD) & $6.20( \pm 8.29)$ & $6.37( \pm 9.61)$ & $5.48( \pm 4.12)$ & 0.751 \\
Mean NLR (SD) & $2.41( \pm 1.09)$ & $2.59 \pm 1.20)$ & $2.01( \pm 0.66)$ & 0.001 \\
Mean PLR (SD) & $145.05( \pm 48.8)$ & $151.14( \pm 51.8)$ & $131.5( \pm 38.8)$ & 0.045 \\
Median PNI (SD) & $44.94( \pm 3.17)$ & $45.13( \pm 2.69)$ & $44.53( \pm 4.06)$ & 0.354 \\
\hline
\end{tabular}

SD: standard deviation; LMR: Iymphocyte-to-monocyte ratio; NLR: neutrophil-to-lymphocyte ratio; PLR: platelet-to-lymphocyte ratio; PNI: prognostic nutritional index.

Table III. RAl, recurrences and lymph nodes recurrence frequency.

\begin{tabular}{lcccc} 
& Population & $\begin{array}{c}\leq 55 \text { years } \\
\text { (according to AJCC stage) }\end{array}$ & $\begin{array}{c}>55 \text { years } \\
\text { (according to AJCC stage) }\end{array}$ & p-value \\
RAl therapy (\%) & $59(50.86)$ & $47(58.8)$ & $12(33.3)$ & 0.01 \\
Lymph node recurrences (\%) & $24(20.68)$ & $18(22.5)$ & $6(16.7)$ & 0.63 \\
Mean months of recurrence (SD) & $22.45( \pm 21.71)$ & $26.44( \pm 23.81)$ & $10.50( \pm 3.50)$ & 0.13 \\
\hline
\end{tabular}

SD: standard deviation; RAl: radioactive iodine.

patients were in TNM stage I, while in the $>55$ year group $13.9 \%$ of patients were in TMN stage II, with a significant difference between groups $(p=0.002)$. This difference is due to staging of the malignancy. The patients in stage II had a T3a, and therefore neoplasm size and age determinate the stage [15].

Table II shows baseline haematological values. Only two values, mean NLR and mean PLR, showed a significant difference between groups. Mean NLR was $2.41(\mathrm{SD} \pm 1.09)$ in entire population, $2.59(\mathrm{SD} \pm 1.20)$ in the $\leq 55$ year group and $2.01(\mathrm{SD} \pm 0.66)$ in the $>55$ year group $(\mathrm{p}=0.001)$. Mean PLR was 145.05 (SD \pm 48.8 ) in the entire population, $151.14(\mathrm{SD} \pm 51.8)$ in the $\leq 55$ year group and 131.5 $(\mathrm{SD} \pm 38.8)$ in the $>55$ year group $(\mathrm{p}=0.045)$.
Table III shows data from patients who underwent RAI therapy, lymph node recurrence and DFS in months. The percentage of lymph node recurrence and DFS in months showed no significant differences between groups. In all, 47 patients underwent RAI therapy $(58.8 \%)$ in the $\leq 55$ year group and 12 patients $(33.3 \%)$ in the $>55$ year group with a significant difference between groups $(\mathrm{p}=0.01)$. Our data showed that the indication to RAI was common in young patients.

Table IV shows prognostic scores to evaluate recurrence risk factors; in particular, adjusted NLR showed a HR of $1.828(\mathrm{p}=0.019)$. Other haematological values showed no significant differences in the HR.

We calculated the receiver operating characteristic

Table IV. Univariate and multivariate analysis evaluating risk factors affecting recurrences.

\begin{tabular}{lcccc} 
& Unadjusted HR (95\% CI) & p-value & Adjusted HR $\left.\mathbf{~}^{(95 \%} \mathbf{C l}\right)$ & p-value \\
PNI & $1.110(0.985-1.252)$ & 0.088 & $1.088(0.966-1.225)$ & 0.162 \\
NLR & $1.240(0.873-1.760)$ & 0.229 & $1.828(1.103-3.029)$ & 0.019 \\
LMR & $1.008(0.961-1.057)$ & 0.739 & $1.011(0.958-1.066)$ & 0.696 \\
PLR & $0.997(0.998-1.005)$ & 0.47 & $0.988(0.975-1.001)$ & 0.079 \\
\hline
\end{tabular}

${ }^{a}$ Adjusted for age, full blood count values, procedure and dimension of lesion. HR: hazard ratio; Cl: confidential interval; PNI: prognostic nutritional index; NLR: neutrophil-tolymphocyte ratio; LMR: Iymphocyte-to-monocyte ratio; PLR: platelet-to-lymphocyte ratio. 


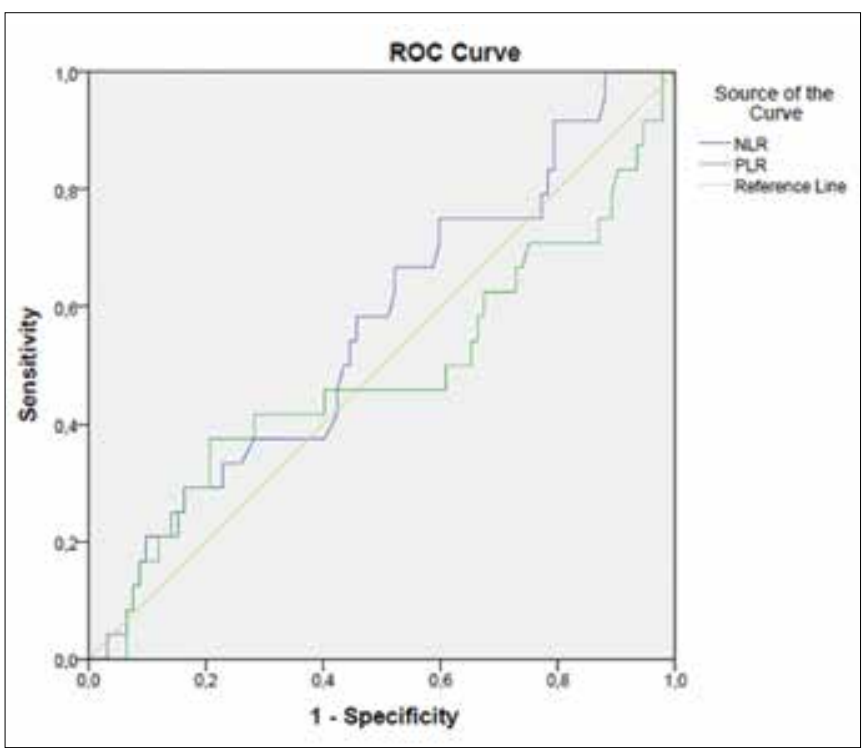

Figure 2. ROC curve of LMR with a cut-off value of 4.635 (AUC 0.540 \pm 0.066 , $66.7 \%$ sensitivity, $48.9 \%$ specificity) and PNI (AUC $0.417 \pm 0.079$ ). (LMR: lymphocyte-to-monocyte ratio; PNI: prognostic nutritional index; AUC: area under curve).

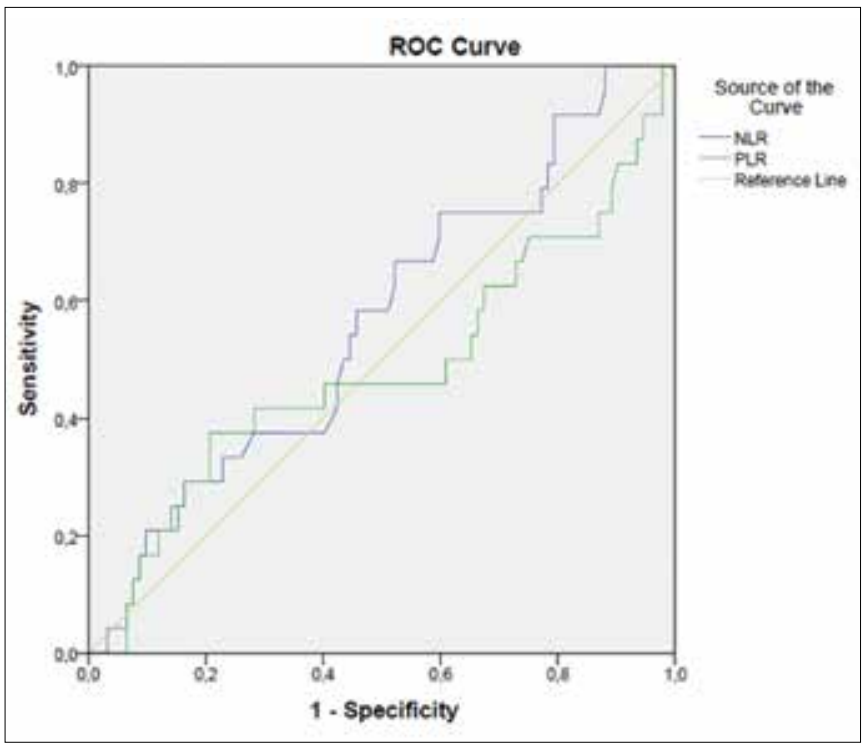

Figure 3. ROC curve of NLR with a cut-off value of 1.750 (AUC $0.564 \pm 0.065$, $75 \%$ sensitivity, $40.2 \%$ specificity) and PLR and (AUC $0.484 \pm 0.075$ ). (NLR: neutrophil-to-lymphocyte ratio; PLR: platelet-to-lymphocyte ratio; AUC: area under curve).

(ROC) curves for LMR and PNI values (Fig. 2). We found 4.635 as best cut-off value for LMR with $66.7 \%$ sensitivity, $48.9 \%$ specificity and an area under the curve (AUC) of $0.540 \pm 0.066$. We did not find a cut-off value for PNI. All PNI values entered in the ROC curve were below the reference line with an area under the curve of $0.417 \pm 0.079$.

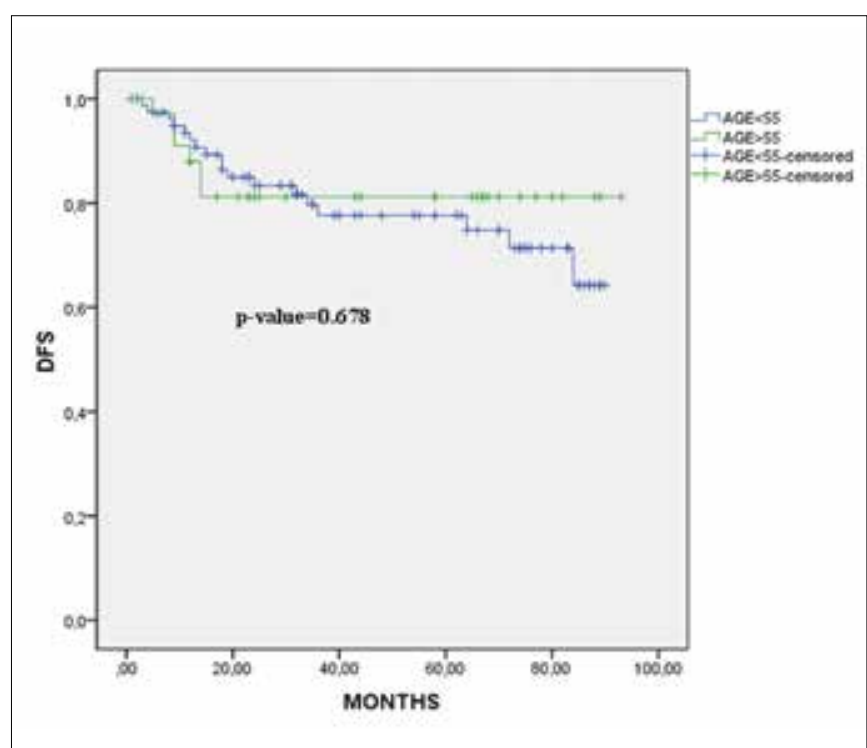

Figure 4. DFS functions distinguished between patients $\leq 55$ years and $>55$ years. DFS was $74.995 \pm 3.236$; DFS was $72.249 \pm 3.689$ in patients $\leq 55$ and DFS was $77.514 \pm 5.717$ in patients $>55$ years. Log-rank test was used to compare recurrences ( $p=0.678)$. (DFS: disease-free survival).

We also calculated a ROC Curve for NLR and PLR (Fig. 3). NLR cut-off values was 1.750 with $75 \%$ sensitivity, $40.2 \%$ specificity and an AUC of $0.564 \pm 0.065$. As for the PNI cut-off value, we found no cut-off value for PLR. All PLR values were below the ROC curve reference line with an AUC of $0.484 \pm 0.075$.

Figure 4 shows DFS curves in the two groups. Overall DFS was $74.995 \pm 3.236$; DFS in the $\leq 55$ year group was $72.249 \pm 3.689$ and in the $>55$ year group was $77.514 \pm 5.717(\mathrm{p}=0.678)$. There was no significant difference between recurrences based on age, as shown above.

\section{Discussion}

Herein, we tried to find a correlation between haematological inflammation indexes and prognosis and risk of recurrence. This is the first study that has examined correlations between LMR, NLR, PLR, PNI and the risk of recurrence in patients with low-risk DTC. The 2015 ATA guidelines suggest undergoing HT in low-risk DTC patients and to undergo NTT/TT in case of expected postoperative RAI therapy, follow-up based on increased serum $\mathrm{Tg}$ and patient preference ${ }^{1}$. Various studies have documented a recurrence rate of $1-2 \%$ in patients who underwent thyroid surgery without postoperative RAI therapy over a followup of 5-10 years ${ }^{13,14}$. Indeed, with a low risk of recurrence, the risk stratification changes during follow-up ${ }^{1}$. The aim of our study was to find an instrument that can identify, at 
the time of diagnosis, patients at higher risk of recurrence which would allow improving the follow-up protocols and plan tailored treatment.

Blood inflammatory cells play an important role in initiation, progression, metastasis and prognosis in oncological patients. Inflammatory blood mediators include neutrophils, lymphocytes, monocytes and platelets. Monocytes and lymphocytes play an important role as anti-tumoural mediators. Low levels of lymphocytes and monocytes has been observed in advanced stage neoplasms and are associated with a poor prognosis ${ }^{15}$. Monocytes penetrate the tumour mass, reduce angiogenesis and induce apoptosis of cancerous cells, thus reducing cancer invasion and progression ${ }^{15,16}$. In the literature, low LMR has been associated with poor prognosis in numerous solid cancers and is an independent prognostic factor. This alteration of the immune system promotes angiogenesis, progression and tumour invasion ${ }^{17,18}$. In thyroid carcinoma the low LMR value is associated with worse prognosis, a high risk of recurrence of ATC and high-risk DTC ${ }^{19,20}$. At the moment no study has examined whether a low LMR value in low-risk DTCs can be an indicator of worse prognosis and increased risk of lymph node recurrence. In our study, the LMR cut-off value was 4.635 with $66.7 \%$ sensitivity and $48.9 \%$ specificity. In the literature, various studies have reported that NLR is an independent prognostic factor in numerous solid tumours ${ }^{5,21}$. However, there are few in DTC. A high ratio is due to an increased neutrophil count compared to lymphocyte count. As mentioned above, neutrophils produce VEGF and inhibit TNF- $\alpha$, facilitating tumour progression and invasion, and a decreased lymphocyte and monocyte count is associated with reduction in antineoplastic activity ${ }^{4,15,16}$. Moreover, neutrophils promote the secretion of IL-2, IL-6 and IL-8, contributing to tumourigenesis ${ }^{4}$. Despite this, the exact correlation between NLR and cancer prognosis still is not entirely clear. Chen et al. suggested that a low NLR $(\leq 1.6)$ is associated with a high risk of PTC recurrence, but with a follow-up of $12.2 \pm 3.8$ months it is not possible to have an estimate of DFS and OS at 5 or 10 years ${ }^{22}$. Ceylan et al. suggested an NLR cut-off value of 1.92 and a correlation between a high NLR, large tumour size and positivity of extra-thyroidal spread ${ }^{23}$. Seretis et al. reported that an elevated preoperative mean NLR is present in patients with microcarcinoma and in DTC, and found that elevated mean NLR does not show any significant difference between patients with multifocal or unifocal microcarcinoma. They did not evaluate an NLR cut-off, but only the NLR preoperative mean ${ }^{24}$. In the literature there are no univocal and concordant data, and thus further studies are needed to find possible correlations between inflammatory indexes and risk of DTC lymph node recurrence to validate them as independent risk factors. In our population, we found an NLR cut-off value of 1.750 with $75 \%$ sensitivity and $40.2 \%$ specificity. This suggests that we have a good chance of finding unwell patients with NLR values $\geq 1.750$, but a poor chance of finding healthy patients. Therefore, only NLR showed a valid HR for recurrence, and indeed it is associated with almost twice the risk of recurrence (Adjusted HR 1.828, $\mathrm{p}=0.019$ ), while patient age showed no significant difference in terms of risk of recurrence. Even if patients with $>55$ years had higher DFS, there was no significant difference between groups $(\mathrm{p}=0.678)$. This can be explained by the fact that in our cohort there were more patients $\geq 55$ years undergoing RAI therapy.

According to the most recent studies, there are few correlations between PLR and prognosis of patients with DTC. Ceylan et al. found no correlation between clinicopathologic features of PTC and PLR ${ }^{23}$. Ozmen et al. reported that PLR and NLR are more successful indexes for diagnosis and recurrence of DTC than C-reactive protein (they included FTC, PTC and microcarcinoma). They found that high NLR and PLR are associated with high levels of thyroglobulin and worse prognosis, but their study did not evaluate cut-off values of these indexes ${ }^{25}$. In our study, we did not find a cut-off value for PLR because the PLR AUC was $0.484 \pm 0.075$ and all values were under reference line. Therefore, further studies are necessary to correlate PLR with multiple variables.

PNI is a score that assesses the patient's nutritional status and immune status based on evaluation of lymphocyte counts and albumin. PNI is a prognostic independent score in most solid cancers, including colorectal cancer, gastric cancer, malignant pleural mesothelioma, hepatocellular carcinoma, pancreatic cancer and breast cancer. Studies show that a low PNI value is associated with worse prognosis due to the deficiency in the nutritional state and deficits of the immune system ${ }^{9-11}$. In our study, we did not found a cut-off value for PNI. Our hypothesis was that we selected a population without concomitant pathologies and with low-risk DTC, a type of indolent tumour with good prognosis.

This study showed that only NLR was a prognostic score, but further studies are necessary to clarify and validate its specific role.

\section{Conclusions}

The correlation between inflammatory indexes and OS and DFS has been established in numerous solid tumours. In our study, NLR emerged as a prognostic factor. Obviously, additional studies are needed to demonstrate this correlation and cannot yet be used to stage and stratify patients 
with low-risk DTC. The limitation of our study is its retrospective nature. Follow-up should be extended to 10 years to better assess the risk of long-term recurrence and a larger population should be examined. Our hope is that in the future these scores can be used to predict the risk of recurrence and to identify patients with low-risk DTC to be submitted to RAI therapy to avoid delays in treatment and to avoid overtreatment by implementing tailored therapy.

\section{Acknowledgements}

The ethical committee of University of Campania "Luigi Vanvitelli" approved the study protocol: AOU4588/2020. The datasets used and/or analysed during the current study are available from the corresponding author on reasonable request.

\section{References}

1 Haugen BR, Alexander EK, Bible KC, et al. 2015 American Thyroid Association Management Guidelines for adult patients with thyroid nodules and differentiated thyroid cancer: the American Thyroid Association Guidelines Task Force on thyroid nodules and differentiated thyroid cancer. Thyroid 2016;26:1-133. https://doi.org/10.1089/ thy. 2015.0020

2 Gambardella C, Patrone R, Di Capua F, et al. The role of prophylactic central compartment lymph node dissection in elderly patients with differentiated thyroid cancer: a multicentric study. BMC Surg 2018;18(Suppl 1):110. https://doi.org/10.1186/ s12893-018-0433-0

3 Cabanillas ME, McFadden DG, Durante C. Thyroid cancer. Lancet 2016;388:2783-95. https://doi.org/10.1016/S0140-6736(16)30172-6

4 Dupré A, Malik HZ. Inflammation and cancer: what a surgical oncologist should know. Eur J Surg Oncol 2918;44:566-70. https://doi. org/10.1016/j.ejso.2018.02.209

5 Mercier J, Voutsadakis IA. Comparison of hematologic and other prognostic markers in metastatic colorectal cancer. J Gastrointest Cancer 2019;50:493-506. https://doi.org/10.1007/s12029-018-0108-1

6 Liu D, Jin J, Zhang L, et al. The neutrophil to lymphocyte ratio may predict benefit from chemotherapy in lung cancer. Cell Physiol Biochem 2018;46:1595-605. https://doi.org/10.1159/000489207

7 Feng JF, Huang Y, Chen QX. Preoperative platelet lymphocyte ratio (PLR) is superior to neutrophil lymphocyte ratio (NLR) as a predictive factor in patients with esophageal squamous cell carcinoma. World $\mathbf{J}$ Surg Oncol 2014;12:58. https://doi.org/10.1186/1477-7819-12-58

8 Sun G, Li Y, Peng Y, et al. Impact of the preoperative prognostic nutritional index on postoperative and survival outcomes in colorectal cancer patients who underwent primary tumor resection: a systematic review and meta-analysis. Int J Colorectal Dis 2019;34:681-9. https:// doi.org/10.1007/s00384-019-03241-1

9 Migita K, Takayama T, Saeki K, et al. The prognostic nutritional index predicts long-term outcomes of gastric cancer patients independent of tumor stage. Ann Surg Oncol 2013;20:2647-54. https://doi. org/10.1245/s10434-013-2926-5

10 Pinato DJ, North BV, Sharma R. A novel, externally validated inflammation-based prognostic algorithm in hepatocellular carcinoma: the prognostic nutritional index (PNI). Br J Cancer 2012;106:1439-45. https://doi.org/10.1038/bjc.2012.92
11 Wang Y, Battseren B, Yin W, et al. Predictive and prognostic value of prognostic nutritional index for locally advanced breast cancer. Gland Surg 2019;8:618-26. https://doi.org/10.21037/gs.2019.10.08

12 Zanoni DK, Patel SG, Shah JP. Changes in the $8^{\text {th }}$ edition of the American Joint Committee on Cancer (AJCC) staging of head and neck cancer: rationale and implications. Curr Oncol Rep 2019;21:52. https://doi.org/10.1007/s11912-019-0799-x

13 Vaisman F, Shaha A, Fish S, et al. Initial therapy with either thyroid lobectomy or total thyroidectomy without radioactive iodine remnant ablation is associated with very low rates of structural disease recurrence in properly selected patients with differentiated thyroid cancer. Clin Endocrinol (Oxf) 2011;75:112-9. https://doi.org/10.1111/j.13652265.2011.04002.x

14 Durante C, Montesano T, Attard M, et al. Long-term surveillance of papillary thyroid cancer patients who do not undergo postoperative radioiodine remnant ablation: is there a role for serum thyroglobulin measurement? J Clin Endocrinol Metab 2012;97:2748-53. https://doi. org/ 10.1210/jc.2012-1123

15 Chanmee T, Ontong P, Konno K, et al. Tumor-associated macrophages as major players in the tumor microenvironment. Cancers (Basel) 2014;6:1670-90. https://doi.org/10.3390/cancers6031670

16 Goswami KK, Ghosh T, Ghosh S, et al. Tumor promoting role of anti-tumor macrophages in tumor microenvironment. Cell Immunol 2017;316:1-10. https://doi.org/10.1016/j.cellimm.2017.04.005

17 Pan YC, Jia ZF, Cao DH, et al. Preoperative lymphocyte-to-monocyte ratio (LMR) could independently predict overall survival of resectable gastric cancer patients. Medicine (Baltimore) 2018;97:13896. https://doi.org/10.1097/MD.0000000000013896

18 Sun Y, Zhang L. The clinical use of pretreatment NLR, PLR, and LMR in patients with esophageal squamous cell carcinoma: evidence from a meta-analysis. Cancer Manag Res 2018;10:6167-79. https:// doi.org/10.2147/CMAR.S171035

19 Yokota M, Katoh H, Nishimiya H, et al. Lymphocyte-monocyte ratio significantly predicts recurrence in papillary thyroid cancer. J Surg Res 2020;246:535-43. https://doi.org/10.1016/j.jss.2019.09.034

20 Ahn J, Song E, Oh HS et al. Low lymphocyte-to-monocyte ratios are associated with poor overall survival in anaplastic thyroid carcinoma patients. Thyroid 2019;29:824-9. https://doi.org/10.1089/ thy. 2018.0684

21 Szkandera J, Gerger A, Liegl-Atzwanger B, et al. The derived neutrophil/lymphocyte ratio predicts poor clinical outcome in soft tissue sarcoma patients. Am J Surg 2014;210:111-6. https://doi.org/10.1016/j. amjsurg.2014.10.021

22 Chen W, Wei T, Li Z, et al. Association of the preoperative inflammation-based scores with TNM stage and recurrence in patients with papillary thyroid carcinoma: a retrospective, multicenter analysis. Cancer Manag Res 2020;12:1809-18. https://doi.org/10.2147/CMAR.S239296

23 Ceylan Y, Kumanlığlu K, Oral A, et al. The correlation of clinicopathological findings and neutrophil-to-lymphocyte and plateletto-lymphocyte ratios in papillary thyroid carcinoma. Mol Imaging Radionucl Ther 2019;28:15-20. https://doi.org/10.4274/mirt.galenos.2018.60490

24 Seretis C, Gourgiotis S, Gemenetzis G, et al. The significance of neutrophil/lymphocyte ratio as a possible marker of underlying papillary microcarcinomas in thyroidal goiters: a pilot study. Am J Surg 2013;205:691-6. https://doi.org/10.1016/j.amjsurg.2012.08.006

25 Ozmen S, Timur O, Calik I, et al. Neutrophil-lymphocyte ratio (NLR) and platelet-lymphocyte ratio (PLR) may be superior to C-reactive protein (CRP) for predicting the occurrence of differentiated thyroid cancer. Endocr Regul 2017;51:131-6. https://doi.org/10.1515/enr2017-0013 